\title{
Super-radiative self-compression of photo-injector electron bunches and the use of this effect for realization of a THz source based on spontaneous coherent emission from a short dense electron bunch
}

\author{
Andrei Savilov $^{1,2}$, I.V. Bandurkin ${ }^{1}$, Yu.S. Oparina ${ }^{1,2}$ \\ ${ }^{1}$ Institute of Applied Physics, N.Novgorod, Russia, savilov@appl.sci-nnov.ru \\ ${ }^{2}$ Lobachevski State University of Nizhny Novgorod, N.Novgorod, Russia
}

\begin{abstract}
Laser-driven photo-injectors allow formation of compact and accessible sources of dense electron bunches with a moderate energy of 3-6 MeV, picosecond pulse durations, and charges of up to $1 \mathrm{nC}$ and even greater. These bunches can be used for realization of comparatively simple and compact terahertz sources operating in the regime of spontaneous coherent undulator radiation. This type of radiation is realized, when the effective axial length of bunches is shorter than the radiation wavelength, so that the wave packets emitted by each of the electrons add up basically in phase. The $\mathrm{THz}$ source based on the spontaneous emission has a number of advantages as compared to the more traditional freeelectron laser (FEL) based on the undulator emission induced due to bunching of a long electron beam by the radiated wave. These are a relatively high efficiency of the emission from the "ready-for-radiation" bunch, a narrow frequency band of the radiated rf signal in a system without a feedback system and an input rf signal, and the phase of the radiated rf signal fixed by the electron bunch phase. These advantages make the $\mathrm{THz}$ source based on the spontaneous emission attractive for a number of important applications, including the use of synchronization of such an oscillator with the X-ray FEL in pump-probe experiments.
\end{abstract}

An example of the THz source based on the spontaneous emission is described in $[1,2]$; a $0.3 \mathrm{nC}$ $5 \mathrm{MeV}$ e-bunch with the $0.1 \mathrm{~mm}$ initial length propagating in an undulator with a period of $2.5 \mathrm{~cm}$ can generate a 20 ps narrow-band rf pulse at a frequency close to $2 \mathrm{THz}$ with a power of tens MW; the efficiency of this process can be over $10 \%$.

The spontaneous emission occurs while the bunch phase size is smaller than $2 \pi$, so that wave packets emitted by each of the electrons add up basically in phase. In the case of the ubitron (as well as Cherenkov) source, this means that the bunch length must stay shorter than the radiation wavelength during the whole radiation region. Therefore, realization of a $\mathrm{THz}$ source based on the spontaneous coherent radiation requires (1) creation of short enough $(0.3 \mathrm{~mm}$ or shorter) dense electron bunches and (2) the presence of a mechanism of stabilization of the phase size of the bunch with respect to the radiated wave. The latter is especially important, taking into account the axial Coulomb repulsion, which is very strong in short dense photo-injector bunches and lead to a significant spreading of the bunch during its motion through a path being as short as few decimeters.

In this work, we propose and study a radiative mechanism for compression and control the axial size of the bunch. This is the axial compression under effect of the spontaneous super-radiation of a long-wavelength wave. In fact, we propose a bicolor (two-wave) rf source operating in the regime of spontaneous coherent undulator radiation (Fig. 1).

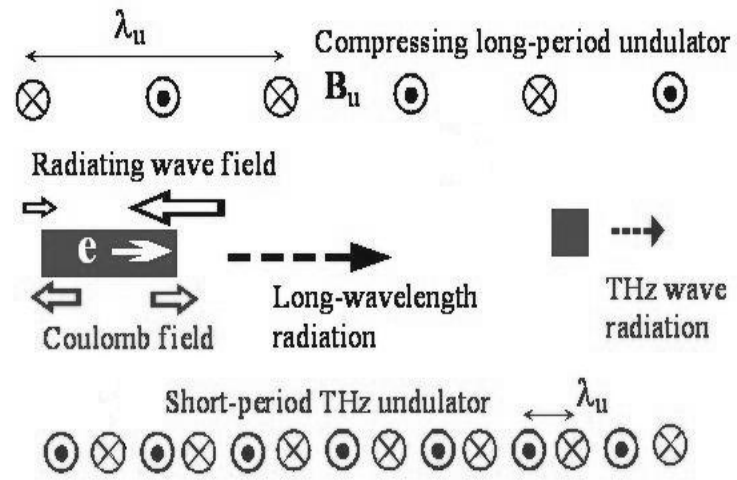

Fig. 1. Bicolor rf source: spontaneous radiation of longwavelength wave leads to the axial compression of the electron bunch and to the spontaneous excitation of the $\mathrm{THz}$ wave

The use of an auxiliary undulator with a relatively long period leads to the super-radiation of a wave with a long wavelength (longer than the initial axial size of the bunch). In certain conditions, the electric field of this auxiliary wave possesses the "proper" phase with respect to the bunch, namely, the bunch front is placed in the maximum of the decelerating phase of this wave, whereas the bunch tail is close to the zero field (Fig. 2). In this situation, the auxiliary long-wavelength wave can provide a significant axial compression of the bunch even in the case of a high charge, when the Coulomb repulsion is strong. The bunch compressed down to a "terahertz" length can radiate the $\mathrm{THz}$ signal in the main short-period undulator.

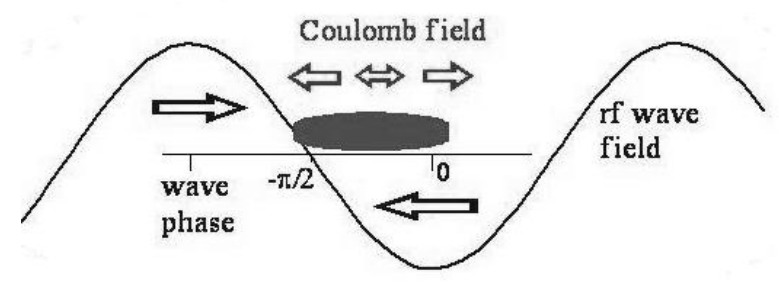

Fig. 2. The bunch phase with respect to the radiated long-wavelength wave

A principal point is the use of the regime of grazing of dispersion characteristics, when the group velocity of the resonant wave coincides with the axial electron velocity, $v_{g r}=v_{z}$. In this case, the regime of the rf wave emission has super-radiative character; this means that the radiated $\mathrm{rf}$ wave does not do not leave the region of the 
electron bunch. The use of this super-radiation regime provides both a high intensity of the compressing rf field and the "proper" phase of this field (Fig. 3).
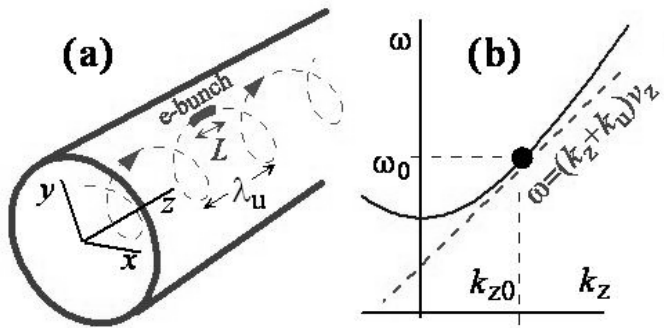

Fig. 3. (a) Electron bunch moving along a helical undulator. (b) Dispersion diagram of the operating waveguide mode

According to a quasi-analytical self-consistent theory describing the motion of a short electron bunch in the radiated wave field and the Coulomb field created by the bunch, the self-compression effect takes place, if the bunch charge is small enough. The permitted charge is determined by the following formula:

$$
\frac{I}{I_{A}} \times \frac{N_{w}^{4}}{K_{u}^{8}} \approx\left(\frac{R}{L_{0}}\right)^{5} \gamma^{-4} .
$$

Here, $I=Q c / L_{0}$ is the electron current, $L_{0}$ is the initial bunch length, $K_{u}$ is the undulator factor, $N_{w}$ is the norm of the excited transverse waveguide mode, $I_{A}=m c^{3} / e, R$ is the beam radius, and $\gamma$ is the initial relativistic Lorants-factor of electrons.
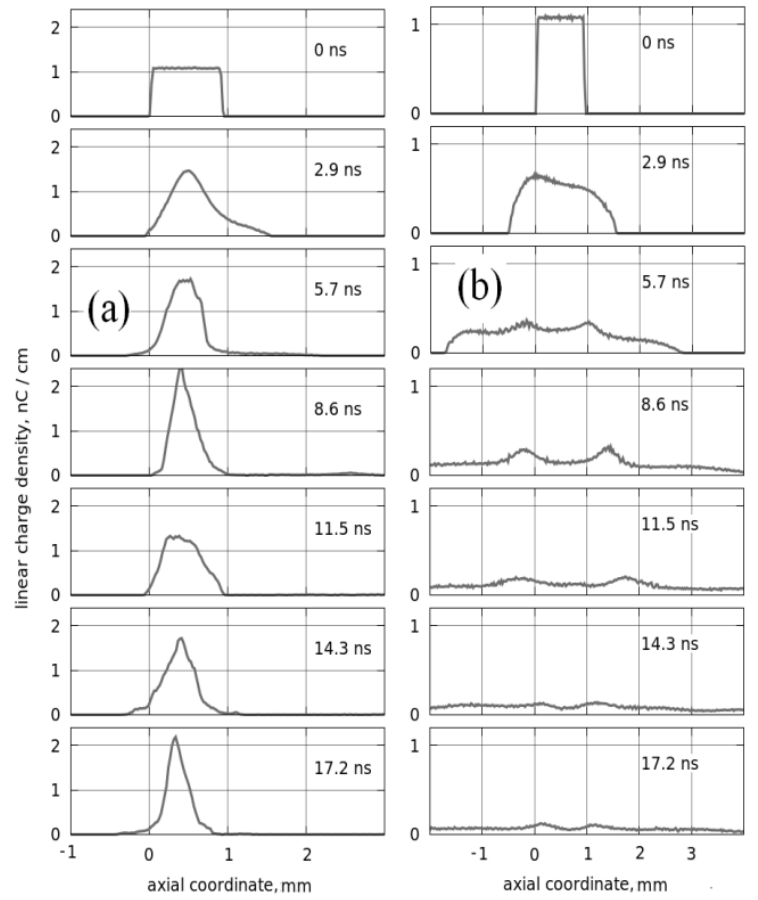

Fig. 4. Evolution in time of the axial distribution of the charge density an electron bunch with initial length $0.9 \mathrm{~mm}$ in (a) the super-radiative case (when the undulator period $6 \mathrm{~cm}$ corresponds to the group resonance condition), and in (b) the case of slightly shorter $(5 \mathrm{~cm})$ undulator period, when the group resonance condition is not fulfilled
Let us consider a bunch with the length $L_{0}=0.9 \mathrm{~mm}$ ( 3 ps). If the super-radiation of the auxiliary longwavelength wave in the bicolor source shown in Fig. 1 provides the axial compression of this bunch by a factor of $1 / 5$, then the compressed bunch length is $L=0.18 \mathrm{~mm}$, which is enough to provide spontaneous radiation of the operating short-wavelength wave at a frequency of $1 \mathrm{THz}$. At $R=1 \mathrm{~mm}$ and $K_{u}=0.7$ this leads to a $3 \mathrm{ps}$ bunch charge $Q \approx 170 \gamma^{-4}$ nC. For instance, at $\gamma=7$ $(3 \mathrm{MeV})$ the bunch charge is close to $0.1 \mathrm{nC}$. In this case, the auxiliary long-wavelength wave frequency $\lambda=$ $1.8 \mathrm{~mm}$, and the proper group velocity of $\mathrm{TE}_{1,1}$ waveguide mode $\left(v_{g r}=v_{z}\right)$ is provided at the waveguide diameter $\approx 6 \mathrm{~mm}$. The undulator period amounts $\approx 6 \mathrm{~cm}$, and the length of the compressing system amounts 12 meters. At the same time, the permitted bunch charge strongly depends on the bunch transverse size, $Q \propto R^{5}$, so that an increase in the bunch radius up to $R=1.5 \mathrm{~mm}$ should result in an increase of the permitted charge up to $\sim 1 \mathrm{nC}$.

We examine the superradiation compression by means of direct simulations of the example described above on the basis of the code KARAT. This is a fully electromagnetic code based on Particle-in-Cell method and direct solving Maxwell equations in systems with complex geometry. Figure 4 a illustrates the evolution of the axial distribution of the charge density in the electron bunch in the time in this super-radiative case. Simulations predict axial compression of the electron bunch (with a compression factor of $\sim 1 / 5$ ) under effect of the radiated wave field. Just for comparison, Fig. b illustrates the same system with one difference, namely, a slightly shorter undulator period $\left(\lambda_{u}=5 \mathrm{~cm}\right)$, so that the group resonance condition $v_{g r}=v_{z}$ is not provided, and the process of rf wave emission does not possess superradiation properties. Due to this fact, the radiated $\mathrm{rf}$ wave possesses significantly lower intensity as compared to the super-radiation case shown in Fig. 4 a. In fact, in this case electrons move mainly in the Coulomb electric field of the bunch. This results in a fast destruction of the bunch. This proves that the compression observed in the "proper" case is provided under effect of the rf wave field excited in the regime of super-radiation.

The work is supported by IAP RAS Project 00352014-0012, and Russian Foundation for Basic Research Project 16-02-00794 A.

\section{References}

1. N. Balal, I.V. Bandurkin, V.L. Bratman, E. Magory, and A.V. Savilov. Negative-mass mitigation of Coulomb repulsion for terahertz undulator radiation of electron bunches. // Appl. Phys. Lett. 2015. V. 107, P. 163505.

2. Y. Lurie, V.L. Bratman, and A.V. Savilov. Energy enhancement and spectrum narrowing in terahertz electron sources due to negative mass instability // Phys. Rev. AB. 2016. V.19. P. 050704. 